\title{
Nova grade curricular do BCC-IME-USP
}

\author{
Daniel Macêdo Batista ${ }^{1}$, Giuliano Salcas Olguin ${ }^{2}$, Jackson José de Souza $^{1 *}$ \\ José Coelho de Pina ${ }^{1}$, Pedro Paulo Vezzá Campos ${ }^{1 *}$, William Alexandre Miura Gnann ${ }^{1 *}$ \\ ${ }^{1}$ Departamento de Ciência da Computação \\ Instituto de Matemática e Estatística - Universidade de São Paulo \\ São Paulo - SP \\ \{batista, jackson, coelho, pedrovc, gnann\}@ime.usp.br \\ ${ }^{2}$ Faculdade de Educação - Universidade de Campinas \\ Campinas - SP \\ giuliano.olguinegmail.com
}

\begin{abstract}
Resumo. A última reformulação na grade curricular do Bacharelado em Ciência da Computação (BCC) do IME-USP deu-se em 1998. Há quatro anos um grupo de alunos, ex-alunos e professores do BCC iniciou um estudo com o objetivo de propor atualizações para a grade curricular do curso. Entre as principais mudanças propostas por este grupo estão a criação de trilhas e o aumento do número de disciplinas optativas eletivas, permitindo, inclusive, que os alunos cursem mais disciplinas de outras áreas e mesmo de outras universidades. Atualmente o Departamento de Ciência da Computação está implementando essas propostas. Neste texto apresentamos o processo de mudança da grade, a nova grade curricular, bem como as diferenças entre as grades curriculares antiga, vigente e a proposta.
\end{abstract}

\section{Introdução}

Na década de 90 os cursos de graduação em computação eram basicamente de Ciência da Computação (CC), com ênfase em software, e Engenharia da Computação, com ênfase em hardware. No início dos anos 2000 essa divisão deixou de ser bicromática e vários outros cursos surgiram para atender um espectro bem maior de necessidades e de adequação à expansão da computação [CS 2001]. Desde então essa tendência policromática tem continuado [CS 2005]. Novos pensamentos e novas tecnologias tem alterado a compreensão da sociedade acerca dos conhecimentos fundamentais de um cientista da computação.

A dinâmica das mudanças em computação indicam que grades curriculares, ou simplesmente grades, em CC devem ser continuamente monitoradas num ciclo de atualizações. Em conjunto, a grande quantidade de especialidades da computação e a interdisciplinaridade da área sugerem grades que sejam flexíveis e que possam ser rapidamente atualizadas. Dessa forma, inclusive, os estudantes de CC terão a possibilidade de decidir o caminho de estudo a ser trilhado [CS 2013] e se desenvolverem naqueles tópicos que tenham maior interesse e aptidão. Além disso, é importante que o curso forme cidadãos críticos e com consciência.

\footnotetext{
*financiado pelo Programa Ensinar com Pesquisa da Pró-Reitoria de Graduação da USP e pelo IME-USP
} 
Entidades que sugerem modelos de grades para CC como a Association for Computing Machinery (ACM), a Institute of Electrical and Electronics Engineers (IEEE) e a Sociedade Brasileira de Computação (SBC) tem apontado para a importância de diminuir a quantidade de disciplinas obrigatórias, permitindo o surgimento e desaparecimento de disciplinas especializadas que garantam que mudanças nas tendências da computação não tornem uma grade obsoleta [CS 2013]. A relevância de grades flexíveis, não só em cursos de CC, foi um dos tópicos do discurso de posse e em entrevistas do atual reitor da Universidade de São Paulo (USP) [Hebmüller 2014]. Esse também foi o tema de um simpósio organizado pela Fundação de Amparo à Pesquisa do Estado de São Paulo (FAPESP) [Alisson 2014] no início de 2014. A principal característica da nova grade que está sendo proposta para o BCC é a sua flexibilidade.

Relatos de experiência sobre reformulações de grades curriculares tem sido frequentes [Leite et al. 2013, Setti et al. 2014]. Em [Setti et al. 2014], os autores focam na motivação para a mudança curricular, que é a evasão. No nosso caso o foco é no processo, que contou com a participação de diversos docentes, estudantes e egressos do BCC. A motivação para a reformulação da grade foram as mudanças constantes na computação. Focando no processo, esperamos que outras universidades possam reproduzí-lo e não simplesmente copiar a nossa grade curricular, já que acreditamos que aspectos locais, tais como o perfil do corpo docente e as expectativas dos alunos, podem afetar o processo em cada lugar. O foco em [Leite et al. 2013] é mais na apresentação da grade e das ênfases. No nosso caso, a nova grade apresenta o que chamamos de núcleo, com as disciplinas mais básicas, porém o aluno ainda precisa cursar a carga horária mínima exigida pelo MEC para obtenção do diploma de Bacharel em Ciência da Computação. Reconhecemos que estudantes de computação sentem-se atraídos por trabalhar antes de terminar o curso mas é desejável que estágios sejam feitos apenas no último ano. A reformulação do BCC é baseada na escolha de trilhas por parte dos alunos, sendo que eles não são obrigados a escolherem uma ênfase para concluir o curso. Todos os detalhes do processo de reformulação estão relatados no documento "Reformulação do BCC-IME-USP" [de Almeida Losnak et al. 2014], que pode ser consultado caso esses detalhes não estejam presentes neste artigo.

\section{Breve histórico e motivação}

A Universidade de São Paulo (USP) é considerada por muitos como uma das boas universidades brasileiras [THE 2015]. O BCC-IME-USP é tido com um dos bons cursos de CC do Brasil [Folha 2014]. Essas posições tornam o BCC-IME-USP referência para outros cursos do Brasil. Por isso compartilhamos aqui o processo realizado na última reforma curricular com a esperança de que isso gere críticas e sugestões que venham a melhorar os cursos de ciência da computação de todo o país.

Em 2014 o BCC-IME-USP completou 40 anos [Representantes de Classe 2014]. Nesse tempo o curso formou pouco mais de 1250 Bacharéis em Ciência da Computação. Segundo uma pesquisa realizada com ex-alunos do BCC em 2012, 86\% trabalham com computação e 13\% não trabalham mais na área [de Almeida Losnak et al. 2014]. Esses bacharéis estão espalhados nas mais diversas atividades. São professores e pesquisadores em universidades, centros de pesquisa e empresas (Facebook, FATEC, Google, IBM Watson, IBM Research - Brasil, ITA, Microsoft, Queen's University, UFABC, UFF, UNICAMP, Universidade Mackenzie, USP, Texas A\&M University, Universidad Adolfo Iba- 
nezm, ...). Alguns egressos também criaram suas próprias empresas (Alora, Caelum, Kekanto, Playax, Scipopulis, ... ). No que diz respeito à modificação da grade do curso, não pretendemos remover a base teórica e a grande fluência em programação, que é apontada como uma das suas qualidades por diversas empresas [Ribeiro-Neto 2015].

A última reforma da grade do BCC ocorreu em 1998 [Ferreira and da Silva 1999]. Desde então a grade do BCC passou por pequenas alterações, apenas duas disciplinas obrigatórias trocaram de semestre e a grade vigente é essencialmente a mesma que foi implementada há dezessete anos. Há cerca de quatro anos, em uma reunião entre alunos e professores foi sugerido que a grade do BCC deveria ser revista. Assim, no início de 2011, a Comissão de Coordenação do BCC $(\mathrm{CoC})$ criou o Grupo Apoio ao BCC com o objetivo de, entre muitos outros, produzir informações e sugestões para auxiliar o conselho do Departamento de Ciência da Computação (DCC) na reformulação da grade. Esse grupo é formado por alunos, ex-alunos e professores, entre estes estão os autores deste texto. Foi então que teve início o processo de elaboração de uma proposta de grade para o BCC.

Neste texto fazemos referências a três grades do BCC-IME-USP: a grade em vigor até 1997, a qual chamamos de grade antiga [BCC 1997]; a grade em vigência a partir de 1998 até hoje, em 2015, a qual nos referimos como grade vigente [BCC 2014]; e finalmente a grade que gostaríamos que, em grande extensão, entrasse em vigor a partir de 2016 e que chamamos de grade proposta [BCC 2016]. Todas essas grades e muitas informações sobre o BCC-IME-USP estão disponíveis na página do BCC em http://bcc.ime.usp.br. As grades detalhadas com todas as suas disciplinas e regras não são apresentadas aqui neste texto por limitação de espaço.

\section{Processo}

A seguir descrevemos alguns dos vários elementos que fizeram parte do processo de elaboração da grade proposta para o BCC. Entre esses elementos estão: avaliações do BCC feitas pelos alunos semestralmente; pesquisa com ex-alunos; reuniões abertas entre alunos e professores; estudo de grades de BCCs do Brasil e de outros países; apresentações de diretrizes curriculares [SBC 2005, CS 2001, CS 2005, CS 2013]; e pesquisa sobre o núcleo de disciplinas para grade. As opiniões de alunos, ex-alunos, professores e uma visão geral dos cursos de CC foram os ingredientes da grade proposta.

\section{Avaliações do BCC}

Desde 2009 é realizado no BCC um processo de avaliação das disciplinas. Esse processo, que foi desenvolvido e implementado pela Escola Politécnica da USP, não tem como objetivo controlar o andamento das aulas ou os docentes. Fundamentalmente o processo promove a discussão sobre a qualidade do BCC (aulas, material didático, integração das disciplinas de um mesmo semestre, entre outros) e detecta eventuais falhas nos conteúdos curriculares e na inter-relação entre as disciplinas dos diversos semestres do BCC. Nesse processo, alunos que se voluntariam a serem representantes de classe (RCs) elaboram um questionário, juntamente com um orientador pedagógico, que é posteriormente respondido pelos alunos do BCC. Depois das respostas serem compiladas, são realizadas reuniões entre RCs, professores, representantes da $\mathrm{CoC}$ e um orientador pedagógico. Com isso foi criado um banco de dados sobre a qualidade de oferecimento das disciplinas e estabeleceu-se uma rotina de discussão de problemas e possíveis soluções. $\mathrm{O}$ 
projeto da grade proposta para o BCC foi enormemente influenciado por essas avaliações e reuniões. Por exemplo, a partir dessas avaliações foi possível constatar a falta na grade vigente de uma disciplina de Vetores e Geometria no primeiro semestre. Essa disciplina ajudaria no aprendizado de Cálculo II e Álgebra Linear que são disciplinas do segundo semestre da grade.

\section{SBC e as grades de BCCs}

Inicialmente, em 2011, a grade vigente foi avaliada sob a ótica das recomendações do Currículo de Referência da SBC [SBC 2005] e seus núcleos. Em seguida, o mesmo foi feito sob a ótica de outros BCCs do Brasil. Com isso, em maio de 2011, foi gerado o documento Currículo de Referência da SBC e a Grade Curricular do $B C C$ [de Almeida Losnak et al. 2014]. A intenção desse documento foi buscar inspiração observando os modelos e ideias de grades adotadas por outros BCCs pelo Brasil. De fato, encontramos várias ideias interessantes e algumas estão presentes na grade proposta.

Por exemplo, a partir desse documento foi possível observar, entre outras coisas, que no que diz respeito a disciplinas obrigatórias, e em relação a outros BCCs, a grade vigente possui: poucos créditos em disciplinas obrigatórias; poucos créditos no núcleo de Contexto Social e Profissional; e muitos créditos (no mínimo 37\% a mais e no máximo $138 \%$ a mais) no núcleo de Matemática. Além disso, o BCC faz parte de um grupo de três a cinco outros BCCs que têm poucos créditos no núcleo Fundamentos da Computação e de Tecnologia da Computação. É evidente que este fato ou sua alteração ao longo dos anos estão relacionados com o perfil do corpo docente.

Levando em consideração os dados levantados por esse documento, alunos e professores do BCC fizeram várias consideração e sugestões que posteriormente foram inseridas no documento [de Almeida Losnak et al. 2014].

\section{Pesquisa com ex-alunos}

Em agosto de 2012 foi iniciado o processo de contatar ex-alunos do BCC para participarem de uma pesquisa [de Almeida Losnak et al. 2014]. Os objetivos da pesquisa foram, dentre outros, receber dos ex-alunos as suas críticas, sugestões e opiniões sobre o BCC para eventuais mudanças curriculares ou criação de disciplinas. Participaram aproximadamente 260 ex-alunos de um total de cerca de 1250.

Os resultados dessa pesquisa tem fornecido elementos para as tomadas de decisões sobre a grade proposta. Por exemplo, na pesquisa os ex-alunos classificam os conhecimentos adquiridos no BCC em termos de quão úteis foram para as suas atividades profissionais. As opções dadas foram muito, mais ou menos, pouco ou nada útil e o resultado geral está a seguir, de acordo com uma divisão em núcleos baseada no Currículo de Referência da SBC [SBC 2005].

\begin{tabular}{|l|r|c|r|r|c|}
\cline { 2 - 5 } \multicolumn{1}{c|}{} & \multicolumn{3}{c|}{ utilidade } & não \\
Núcleo & muito & mais ou menos & pouco & nada & respondeu \\
\hline BCC como um todo & 68,7 & 26,8 & 3,4 & 0,0 & 1,1 \\
Matemática & 32,1 & 37,7 & 26,0 & 3,4 & 0,8 \\
Probabilidade e Estatística & 30,2 & 36,6 & 26,0 & 6,0 & 1,1 \\
Fundamentos da Computação & 59,6 & 28,3 & 10,9 & 0,0 & 1,1 \\
Tecnologia da Computação & 68,7 & 21,5 & 7,2 & 1,1 & 1,5 \\
Física & 4,2 & 18,1 & 35,8 & 40,4 & 1,5 \\
\hline
\end{tabular}




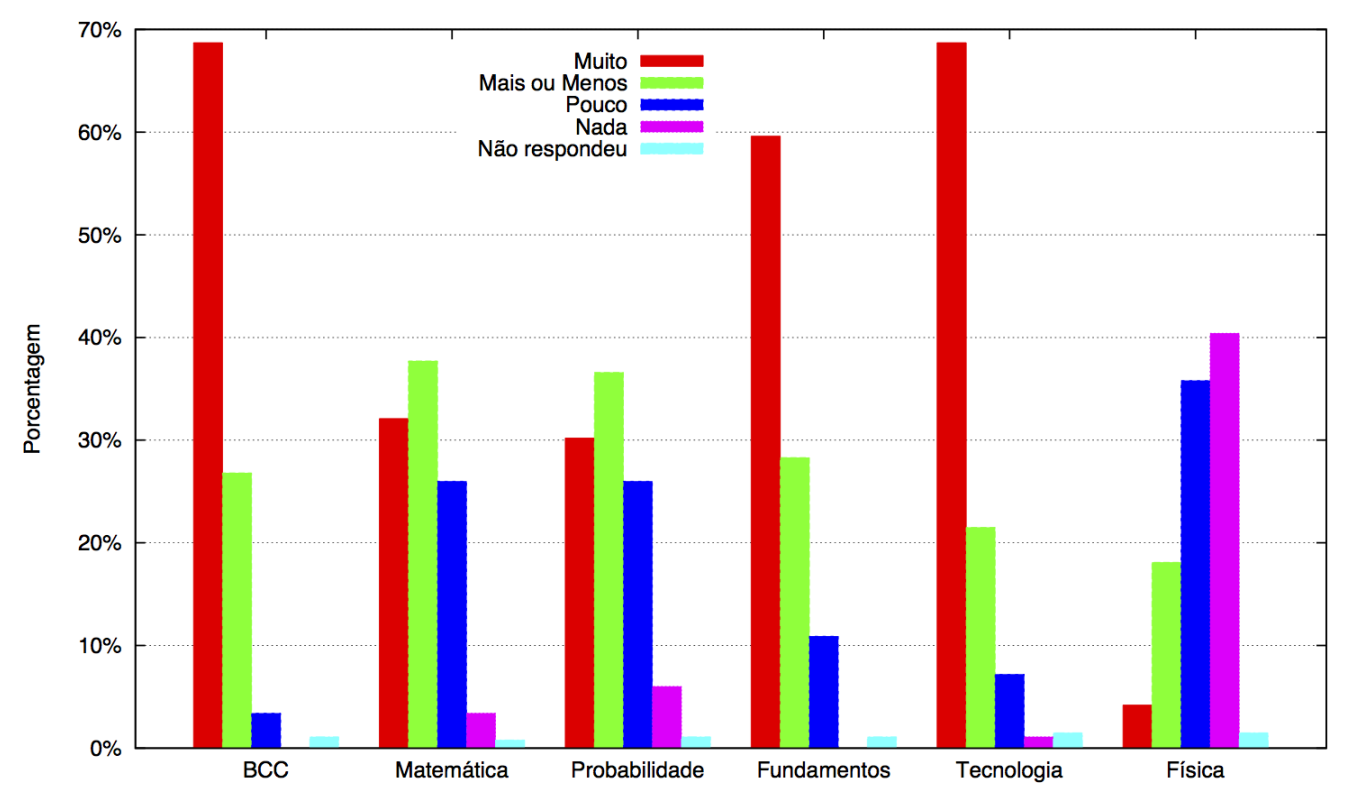

Como exemplo de mais um dos resultados da pesquisa vale mencionar que foi possível observar que ao longo de 40 anos do BCC a disciplina Estrutura de Dados foi considerada relevante para a carreira profissional pela grande maioria dos ex-alunos. Também foi possível observar que determinadas disciplinas foram consideradas mais relevantes pelos ex-alunos de acordo com o período de graduação deles. Isto é esperado tendo em vista a evolução da $\mathrm{CC}$ e as mudanças na grade. Por exemplo, disciplinas consideradas muito relevantes pelos ex-alunos nos anos 70 e 80 foram Projeto de Compiladores e Sistemas Operacionais, no final dos anos 90 foi Sistemas de Bancos de Dados e mais recentemente aparece Laboratório de Programação eXtrema. Dados como esses mencionados e muitos outros enriqueceram sobremaneira a discussão da grade proposta.

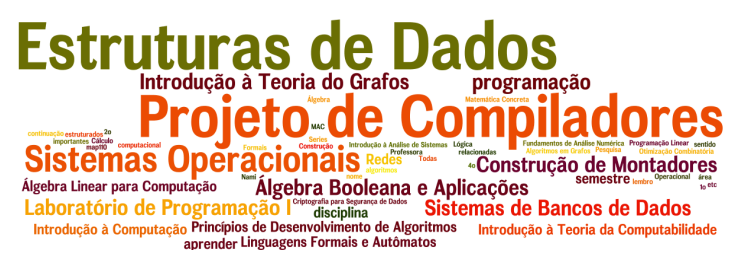

1984-1988

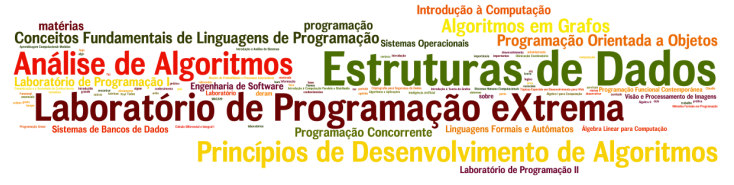

2009-2013

É importante observar que toda a comunidade do BCC-IME-USP, com exceção de funcionários, participou ativamente da definição de metas e no projeto de implementação das ações, que se traduz na nova grade curricular.

\section{Reuniões abertas}

Desde dezembro de 2012 o Grupo Apoio ao BCC tem organizado reuniões para a elaboração de uma proposta de nova grade para o BCC. Essas reuniões ocorreram regularmente, tipicamente a cada duas semanas, das $12 \mathrm{~h}$ às $13 \mathrm{~h}$ e até abril de 2014 foram realizadas aproximadamente trinta reuniões. Acreditamos que o fato das reuniões serem realizadas no horário do almoço e terem a duração fixa de no máximo uma hora colaborou para que elas não se esvaziassem.

Nessas reuniões, para subsidiar as discussões, foram apresentadas várias grades, modelos de currículos, diretrizes curriculares, resultados de pesquisas, sugestões de dis- 
ciplinas, sugestões de modelos de currículos e conversas com professores convidados de outros cursos.

As reuniões foram abertas, amplamente divulgadas e contaram com a participação de alunos dos diversos anos do curso, especialmente os RCs, ex-alunos que são pósgraduandos; e professores. O número de participantes variou entre 10 a 30, dependendo do período do ano. Os relatos de todas as reuniões foram também amplamente divulgados e estão disponíveis a partir da página do BCC.

Vale salientar que nessas reuniões os alunos e ex-alunos tiveram uma participação fundamental. Seus comentários foram muito pertinentes. De fato, eles, mais do que ninguém, têm uma visão geral, ampla e atual do andamento de cada disciplina e do curso com um todo. Informações importantes como relações entre disciplinas listadas como prérequisitos e cumprimento da ementa por parte dos professores são exemplos de dados que fazem parte do dia-a-dia dos alunos e que seriam ignoradas caso alunos não participassem das reuniões.

\section{Pesquisa para determinação do núcleo}

Durante as reuniões abertas foi decidido que, por causa da grande quantidade de especializações possíveis, a grade proposta seguiria um modelo com trilhas de conhecimento. Dessa forma, ficou acordado que um núcleo das disciplinas obrigatórias do curso deveria ser definido. Esse núcleo seria formado pelas disciplinas que os alunos, ex-alunos e professores considerassem relevantes para a maioria das trilhas ou que fossem consideradas indispensáveis na formação de um cientista da computação. Para isto, foram preparados questionários, um para cada par de áreas (knowledge areas) da planilha de habilidades (outcomes) utilizada pela ACM/IEEE no seu projeto de currículo de referência [CS 2013]. Alunos, ex-alunos e professores responderam os questionários. Os resultados dessa pesquisa estão disponíveis na página do BCC.

\section{Objetivos e princípios}

Idealmente, gostaríamos que a grade fornecesse elementos para preparar um cientista da computação responsável, engajado socialmente e consciente da ampla relação da computação com os mais diversos domínios do conhecimento.

Depois de dez reuniões abertas foram apresentados ao conselho do DCC os princípios que norteiam a grade proposta. Dentre esse princípios, os mais fundamentais estão a seguir.

O tamanho do núcleo da grade, formado por disciplinas obrigatórias, deve ser pequeno. Enquanto o leque de tópicos relevantes cresceu, a duração da graduação permaneceu constante. Desta forma, a reformulação deve cuidadosamente escolher os tópicos imprescindíveis a quem quer seguir trabalhando ou estudando na área. Um número pequeno de disciplinas obrigatórias garante que mudanças nas tendências da computação não tornem o currículo defasado rapidamente. Enquanto isso, o surgimento e desaparecimento de disciplinas especializadas garantem que ele permaneça atualizado.

A grade deve ser flexível o suficiente para permitir que os alunos trabalhem em diversas áreas. A CC é um campo vasto que interage e faz uso de diversos conceitos provenientes de matemática, engenharia, psicologia, estatística, artes, linguística, ciências 
naturais, entre outros. Durante a graduação o aluno deve criar uma desenvoltura para trabalhar entre essas diversas disciplinas. Isso pode ser realizado aproveitando a grande variedade e qualidade de disciplinas que as demais unidades da USP oferecem.

Um sistema de trilhas, ênfases ou módulos é interessante para nortear os estudos dos alunos. Este princípio tem como efeito colateral mostrar a riqueza da $\mathrm{CC}$ como área de estudo, tornando a graduação mais atrativa a novos alunos. Cada trilha, ênfase ou módulo deve agrupar as disciplinas relacionadas com uma determinada área da CC de modo a auxiliar o aluno interessado em se especializar naquela área.

\section{Grades antiga, vigente e proposta}

Apesar de estar quase atingindo a maioridade, notamos que a grade vigente tem uma grande qualidade que é a responsável pelo BCC não ter se tornado um curso obsoleto. A grade vigente tem 38 créditos em disciplinas optativas, distribuídos em 32 créditos de optativas eletivas e 6 créditos em optativas livres.

Optativas eletivas são disciplinas dentro de um conjunto preestabelecido, tipicamente disciplinas relacionadas com computação e em sua maioria oferecidas pelo próprio DCC do IME-USP. As optativas livres não tem preestabelecimento do seu conjunto e na grade vigente são, preferencialmente, disciplinas com um caráter de humanidades. Já na grade proposta, optativas livres são livres na acepção da palavra, pois podem ser qualquer disciplina da USP, inclusive da lista de eletivas.

Na USP cada crédito aula corresponde a 50 minutos de aula e cada disciplina tem tipicamente quatro créditos. Assim, na grade vigente o aluno deve cursar usualmente oito disciplinas optativas eletivas e duas optativas livres. Essa carga em disciplinas optativas corresponde a cerca de $20 \%$ do curso. Na grade antiga esse valor era de aproximadamente 16\%. Para 2016 essa porcentagem de disciplinas optativas crescerá para quase $40 \%$. O propósito desse aumento em disciplinas optativas é flexibilizar o BCC.

A seguir apresentamos uma tabela, e o gráfico correspondente, que comparam as grades antiga, vigente e proposta. As disciplinas obrigatórias estão organizadas segundo a classificação de núcleos da SBC [SBC 2005].

\begin{tabular}{|l|r|r|r|r|r|r|}
\cline { 2 - 7 } \multicolumn{1}{c|}{} & \multicolumn{6}{c|}{ grade } \\
\cline { 2 - 7 } \multicolumn{1}{c|}{} & \multicolumn{2}{c|}{ antiga } & \multicolumn{2}{c|}{ vigente } & \multicolumn{2}{c|}{ proposta } \\
\hline Núcleo & créditos & \multicolumn{1}{c|}{$\%$} & créditos & \multicolumn{1}{c|}{$\%$} & créditos & \multicolumn{1}{c|}{$\%$} \\
\hline Créditos em optativas & 27 & 15,9 & 38 & 19,1 & 76 & 39,0 \\
\hline Créditos trabalho & 10 & 5,8 & 24 & 12,1 & 24 & 12,3 \\
\hline Contexto Social e Profissional & 3 & 0,1 & 3 & 0,1 & 7 & 3,6 \\
Matemática & 50 & 29,4 & 50 & 25,1 & 36 & 18,5 \\
Fundamentos da Computação & 36 & 21,2 & 40 & 20,1 & 32 & 16,4 \\
Tecnologia da Computação & 20 & 11,7 & 32 & 16,1 & 16 & 8,2 \\
Ciências básicas & 24 & 14,1 & 12 & 6,0 & 4 & 2,1 \\
Sistemas de Informação & 0 & 0 & 0 & 0 & 0 & 0 \\
\hline Total & 170 & 100 & 199 & 100 & 195 & 100 \\
\hline
\end{tabular}




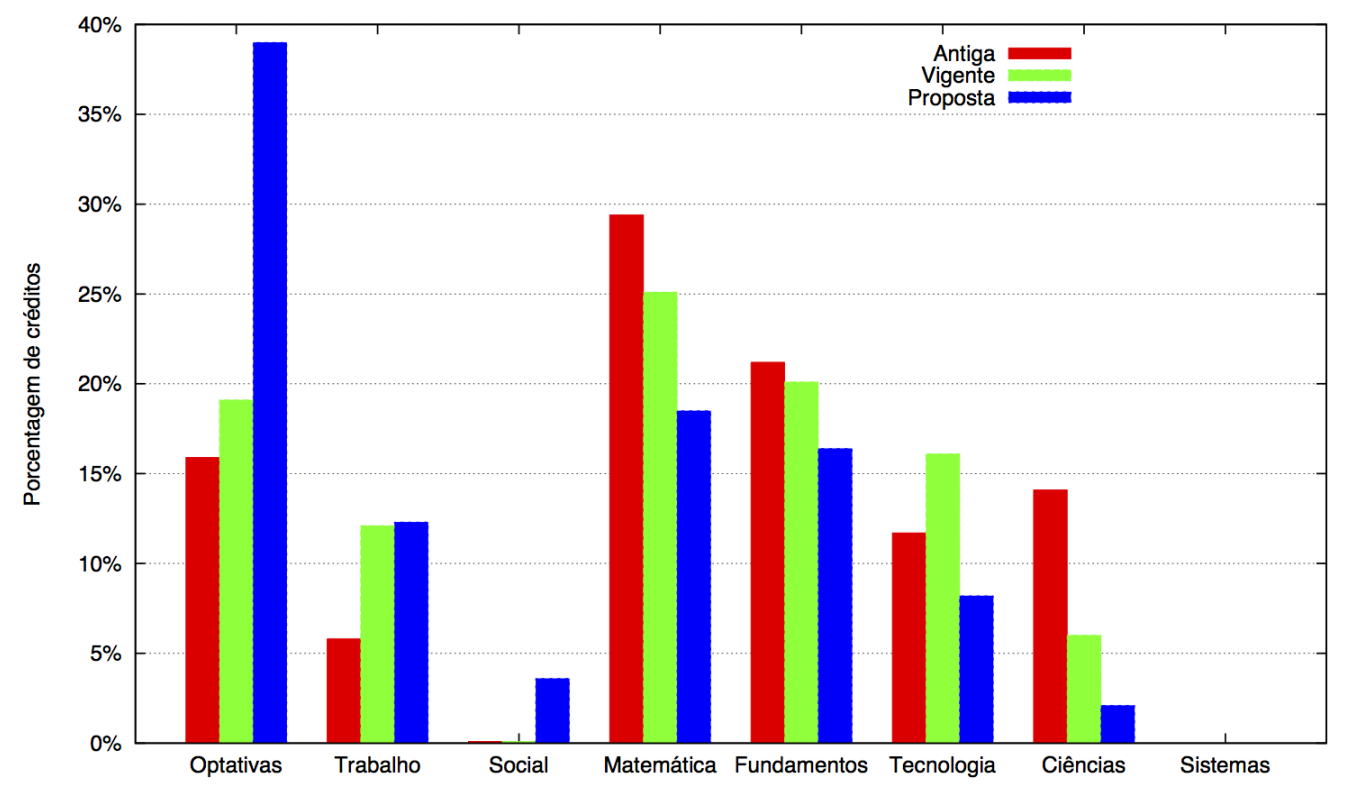

Levando em consideração o número de créditos em disciplinas optativas vê-se que a grade proposta é muito mais flexível que as suas antecessoras. Espera-se com isto dar a oportunidade do aluno entrar em contato com disciplinas de outros cursos como química, física, biologia, ciências sociais, economia, entre outros. Para que este contato se efetive, a CoC deverá trabalhar no sentido das escolas, faculdades e institutos da USP abrirem algumas vagas para alunos do BCC.

Comentamos agora acerca de algumas disciplinas que foram criadas com objetivo de implementar os objetivos e princípios delineados.

A disciplina Introdução ao Bacharelado em Ciência da Computação terá a missão de apresentar o BCC, o IME e a USP aos calouros. Além disso, essa disciplina é um lugar onde os alunos podem constituir grupos de interesse e estender seus horizontes dentro do DCC. Esta disciplina é inspirada nas disciplinas Introdução à Ciência da Computação da Universidade Federal de Minas Gerais, Introdução à Computação da Universidade Federal de Santa Catarina, e Freshmen Immigration Course e Great Theoretical Ideas in Computer Science da Carnegie Mellon University.

Tendo em vista que quase $40 \%$ dos créditos da grade proposta são em disciplinas optativas e o modelo adotado foi o de trilhas, em que os alunos podem seguir diversos caminhos, foi criada a disciplina Caminhos no Bacharelado em Ciência da Computação, com objetivo de no início de segundo ano do $\mathrm{BCC}$ orientar os alunos sobre as várias possibilidades de formação no BCC através de suas trilhas.

A fim de incentivar o desenvolvimento do aluno como indivíduo e cidadão foi criada a disciplina optativa eletiva Atividade Curricular em Comunidade. Esta disciplina é uma reprodução da disciplina Ação Curricular em Comunidade e em Sociedade oferecida pela Universidade Federal da Bahia [UFBA 2013].

Também foram criadas disciplinas optativas para estimular os alunos a se empenharem em alguma atividade independente, como o USPGameDev [USPGameDev 2009] e o Hardware Livre [ArduinoUSP 2013], e terem contato com atividades de pesquisa. São elas a Atividade Curricular em Cultura e Extensão e Atividade Curricular em Pesquisa. 
No que se refere às trilhas, professores do DCC sugeriram até o momento quatro: Algoritmos e Complexidade, Sistemas de Software, Inteligência Artificial e Ciência de Dados. Essas trilhas são fortemente influenciadas pelo perfil do corpo docente do DCC.

\section{Expectativas e lições aprendidas}

Esperamos que a grade proposta facilite a atualização constante do BCC de modo a acompanhar os avanços em CC. Além disso, esperamos também formar cientistas da computação mais responsáveis já que com o modelo de trilhas e a grande quantidade de optativas, os alunos precisarão ser capazes de tomar seus próprios caminhos. Esperamos ainda que a interdisciplinaridade motivada pela grade proposta faça com que os formandos do $\mathrm{BCC}$ colaborem nos avanços da área.

A CoC do BCC deverá trabalhar para que as Escolas, Faculdades e Institutos ofereçam disciplinas livres para os alunos do BCC. Além disso, a CoC deverá estar atenta para possíveis problemas com a grade proposta. Ambas as tarefas deverão ser facilitadas através das Avaliações do BCC que são realizadas em todo semestre.

É importante observar que muitas das nossas expectativas podem não se concretizar. Entretanto, mantendo as avaliações constantes no curso e o contato com ex-alunos, e os empregadores desses ex-alunos, esperamos fazer correções na grade em intervalos de tempo menores do que os dezessete anos para a mudança atual.

Se fôssemos indagados sobre qual foi o componente mais importante em todo esse processo de reformulação da grade, nós responderíamos que foi a participação dos alunos e ex-alunos nas reuniões abertas.

\section{Agradecimentos}

Os nossos mais sinceros agradecimentos aos RCs, alunos, ex-alunos e professores do DCC e de outros departamentos que participaram das reuniões para produzir este projeto de uma nova grade para o BCC. Também somos muito agradecidos ao Programa Ensinar com Pesquisa da Pró-Reitoria de Graduação da USP e o IME-USP pelo apoio financeiro.

\section{Referências}

[Alisson 2014] Alisson, E. (2014). Especialistas defendem currículo mais flexível nas universidades. http://agencia.fapesp.br/18575. Último acesso em 6 de fevereiro de 2014.

[ArduinoUSP 2013] ArduinoUSP (2013). Arduinousp. http: / / social.stoa.usp. br/profile/arduinousp.

[BCC 1997] BCC (1997). Grade curricular do BCC-IME-USP para 1997. http: / / bcc . ime.usp.br/curriculo1997/.

[BCC 2014] BCC (2014). Grade curricular do BCC-IME-USP para 2014. http : / / bcc . ime.usp.br/curriculo2014/.

[BCC 2016] BCC (2016). Grade curricular do BCC-IME-USP para 2016. http: / / bcc . ime.usp.br/curriculo2016/.

[CS 2001] CS (2001). Computing curricula 2001: Computer science. http: //www.acm.org/education/education/education/curric_vols/ cc2001.pdf. Último acesso em 7 de fevereiro de 2014. 
[CS 2005] CS (2005). Computing curricula 2005: The overview report. http://www.acm.org/education/education/curric_vols/ CC2005-March06Final.pdf. Último acesso em 7 de fevereiro de 2014.

[CS 2013] CS (2013). Computer science curricula 2013: Curriculum guidelines for undergraduate degree programs in computer science. http://www.acm.org/ education/CS2013-final-report.pdf. Último acesso em 7 de fevereiro de 2014.

[de Almeida Losnak et al. 2014] de Almeida Losnak, A. L., Caló, A., Batista, D. M., Olguin, G. S., de Souza, J. J., de Pina, J. C., Louro, R. D., and Gnann, W. A. M. (2014). Reformulação do BCC-IME-USP. http://bcc.ime.usp.br/principal/ documentos/reformulacao . pdf. Último acesso em 14 de abril de 2015.

[Ferreira and da Silva 1999] Ferreira, C. E. and da Silva, D. M. (1999). O novo BCC da USP: um Novo Curso para os Desafios do Novo Milênio. In Anais do WEI - XIX Congresso da Sociedade Brasileira de Computação, pages 505-514.

[Folha 2014] Folha (2014). Rank Universitário da Folha 2014. http:// ruf . folha. uol.com.br/2014/rankingdecursos/cienciadacomputacao/.

Último acesso em 10 de abril de 2015.

[Hebmüller 2014] Hebmüller, P. (2014). Reforma do ensino de graduação será meta prioritária. Jornal da USP. http://espaber.uspnet.usp.br/jorusp/?p= 33343. Último acesso em 7 de fevereiro de 2014.

[Leite et al. 2013] Leite, J. C., Gomes, A. V., da Silva, B. S., and do Rego, J. I. (2013). O Curso de Tecnologia da Informação na UFRN. In Anais do WEI - XXXIII Congresso da Sociedade Brasileira de Computação, pages 701-710.

[Representantes de Classe 2014] Representantes de Classe (2014). 40 anos do BCC-IMEUSP. http://bcc.ime.usp.br/40anos/. Último acesso em 10 de abril de 2015.

[Ribeiro-Neto 2015] Ribeiro-Neto, B. (2015). IME: Celeiro de talentos em Computação. http://www.ime.usp.br/images/arquivos/aconteceime/ acontecenoime_edicao25.pdf. Último acesso em 14 de abril de 2015.

[SBC 2005] SBC (2005). Currículo de referência - CC e EC. http://www. sbc . org. $\mathrm{br} /$.

[Setti et al. 2014] Setti, M. G., Emer, M. C. F. P., Amaral, M. A., Merkle, L. E., and Gonçalves, M. M. (2014). Proposta de Flexibilização Curricular do Curso de Sistemas de Informação ofertado pela UTFPR-Curitiba. In Anais do WEI - XXXIV Congresso da Sociedade Brasileira de Computação, pages 1366-1375.

[THE 2015] THE (2015). World Reputation Rankings 2015. http://www. timeshighereducation.co.uk/world-university-rankings/ 2015 /reputation-ranking/. Último acesso em 10 de abril de 2015.

[UFBA 2013] UFBA (2013). Ação Curricular em Comunidade e em Sociedade (ACCS). http: / / www.proext.ufba.br/accs.

[USPGameDev 2009] USPGameDev (2009). Uspgamedev. http://uspgamedev . org/. 